\title{
Enfermedad renal diabética: de cara a la prevención, diagnóstico e intervención temprana
}

\author{
Diabetic kidney disease: from face to prevention, diagnosis and early intervention.
}

\author{
Jorge Eduardo Rico Fontalvo ${ }^{1,2}$ \\ 'Asociación Colombiana de Nefrología e Hipertensión Arterial, comité Nefrodiabetes, Bogotá D.C., Colombia. \\ ${ }^{2}$ Academia de Medicina de Medellín, Medellín, Colombia.
}

$\mathrm{E}$ 1 número de pacientes con diabetes en el mundo entero aumenta cada día. Según la Federación Internacional de Diabetes, para el año 2019 se reportaron alrededor de $32 \mathrm{mi}-$ llones de casos de pacientes diabéticos en Centro y Suramérica (lo que implica una prevalencia promedio del $9,4 \%$ ) y esta cifra puede incrementarse a más de 49 millones en el año $2045^{1}$.

Una de las complicaciones crónicas y más frecuentes que presentan los pacientes diabéticos es la enfermedad renal diabética (ERD), una patología cuya incidencia se ha duplicado en la última década debido, principalmente, al aumento en el número de casos de pacientes que padecen de diabetes ${ }^{2}$. En la actualidad se estima que cerca del 25-35\% de quienes son diagnosticados con diabetes tipo 2 ya tienen complicaciones microvasculares y que a menudo hay un retraso de 4 a 7 años entre el inicio de la enfermedad y su diagnóstico .

Es por eso que la detección temprana, la estadificación y el seguimiento de la progresión de la ERD son los pilares más importantes y la estrategia más costo-efectiva para prevenir y/o retrasar el deterioro de la función renal en pacientes con diabetes tipo 2 y función renal normal o levemente comprometida. En este sentido, se recomienda que al momento del diagnóstico de diabetes tipo 2, y luego cada año o cada 5 años para diabetes tipo 1, se haga una búsqueda activa de ERD.

Los factores de riesgo relacionados con el desarrollo y la progresión de la ERD pueden ser modificables (mal control glucémico, hipertensión arterial, nivel de albuminuria, obesidad, dislipidemia, tabaquismo y presencia de otras complicaciones microvasculares) y no modificables (género masculino, edad avanzada, factores genéticos, raza y tiempo de duración de la diabetes) $)^{3}$.

Definir el riesgo cardiovascular en el paciente diabético hace parte de las estrategias de gestión e intervención temprana, pues el solo hecho de tener diabetes hace que el paciente sea mínimo de riesgo moderado; en otras palabras, no se recomienda hablar de pacientes diabéticos tipo 2 de bajo riesgo cardiovascular. Además, la mayoría de pacientes diabéticos con compromiso renal son de riesgo alto o muy alto.

De igual forma, es importante establecer el riesgo de falla cardíaca en los pacientes con ERD, para lo cual existen varias escalas, además se sabe que la sola presencia de albuminuria, sobre todo cuando su valor es $>300 \mathrm{mg}$ (albuminuria A3), aumenta el riesgo de falla cardiaca ${ }^{3}$.

El tratamiento de los pacientes con ERD debe ser integral y bajo una visión holística de su enfermedad, además debe tener metas y objetivos claros y un abordaje centrado en el paciente y, en mi opinión, en el que se perfile siempre al paciente.

Está comprobado que las intervenciones no farmacológicas mejoran el control metabólico y reducen la presión arterial (PA) y que las farmacológicas controlan la glucemia, la HBA1c, los lípidos, la PA, la hiperuricemia, entre otras ${ }^{4}$.

Citación: Rico Fontalvo J. Enfermedad renal diabética: de cara a la prevención, diagnóstico e intervención temprana. 2020;7(2). https://doi.org/10.22265/acnef.7.2.506. Correspondencia: Jorge Eduardo Rico Fontalvo, jorgericof@yahoo.com 
El control glucémico es fundamental porque está demostrado que con una adecuada intervención se reduce la progresión de las complicaciones microvasculares, dentro de estas la ERD, razón por la cual en los últimos años han surgido terapias farmacológicas innovadoras para el tratamiento de este tipo de pacientes. Los nuevos medicamentos antidiabéticos han demostrado en los estudios de seguridad cardiovascular que reducen el desenlace cardiovascular combinado, el deterioro de la función renal y el riesgo de hospitalizaciones por insuficiencia cardíaca 5 .

En la actualidad están en curso varios estudios prospectivos multicéntricos que tienen como objetivo primario evaluar desenlaces renales en pacientes diabéticos. Uno de estos estudios es el DAPA-CKD, que se desarrolla con dapaglifozina e incluye pacientes diabéticos y no diabéticos con compromiso renal; esta investigación fue finalizada prematuramente por los resultados a favor. Otros estudios son el EMPAKIDNEY, que emplea empaglifozina, y el FLOW, que utiliza semaglutida ${ }^{6}$.

Por otro lado, desde hace aproximadamente 2 años, con el apoyo y aval de la junta directiva de la Asociación Colombiana de Nefrología, un grupo de especialistas decidimos crear y organizar el comité de Nefrodiabetes, el cual está conformado por 11 nefrólogos de diferentes ciudades del país, todos líderes de opinión y con una amplia experiencia en los temas de diabetes y enfermedad renal. Como grupo, hemos participado en varios eventos a nivel regional, nacional e internacional; en julio del presente año comenzamos con la elaboración de las primeras guías colombianas de práctica clínica sobre diagnóstico y tratamiento de ERD, que esperamos estén terminadas en el mes de diciembre, y en la actualidad nos encontramos organizando el primer simposio nacional sobre ERD que se llevará a cabo en el primer semestre del próximo año.
En síntesis, los nefrólogos debemos ser líderes y protagonistas en el manejo integral del paciente diabético, pioneros en la gestión e intervención temprana de riesgo y proactivos en las estrategias de prevención de riesgos en los pacientes diabéticos para que de esta manera podamos ver resultados. En este sentido, nuestro gran objetivo es mejorar el pronóstico de los pacientes diabéticos y, por ende, mejorar su calidad de vida. Por último, como suelo terminar estas columnas y como siempre ha dicho mi padre, recalco que debemos ser protagonistas del desarrollo y no cómplices del subdesarrollo.

\section{Referencias}

1. International Diabetes Federation (IDF). IDF Diabetes Atlas 2019. $9^{\text {th }}$ ed. Belgium: IDF; 2019.

2. Lopera-Vargas JM, Rico-Fontalvo JE, Melgarejo E, CastilloBarrios GA, Ramírez-Rincón A, Gómez AM, et al. Efectos de terapias farmacológicas para el control glucémico en pacientes con diabetes mellitus tipo 2 en los desenlaces vasculares. Rev Colomb. Nefrol. 2020,7(1):44-59.

https://doi.org/10.22265/acnef.7.1.372.

3. Rico-Fontalvo JE. Nefropatía diabética. En: Restrepo CA, Buitrago CA, Torres J, Serna J, editores. Nefrología Básica 2. 2da ed. Bogotá D.C.: Asociación Colombiana de nefrología; 2012.

4. Li J, Albajrami O, Zhuo M, Hawley C-E, Paik JM. Decision Algorithm for Prescribing SGLT2 Inhibitors and GLP-1 Receptor Agonists for Diabetic Kidney Disease. Clin J Am Soc Nephrol. 2020. https://doi.org/10.2215/CJN.02690320.

5. Lin YC, Chang YH, Yang SY, Wu KD, Chu TS. Update of pathophysiology and management of diabetic kidney disease. J Formos Med Assoc. 2018;117(8):662-75. https://doi.org/10.1016/j.jfma.2018.02.007.

6. American Diabetes Association. 6. Glycemic Targets: Standards of Medical Care in Diabetes-2020. Diabetes Care. 2020;43(Suppl 1):S66-S76. https://doi.org/10.2337/dc20-S006. 